www.parjournal.net

\title{
Proximal femur reconstruction using a vascularized fibular epiphysis within a cadaveric femoral allograft in a child with Ewing sarcoma: a case report
}

\author{
Michelle Y. Seu ${ }^{1}$, Allison Haley ${ }^{1}$, Brian H. Cho ${ }^{1}$, Hannah M. Carl'1, Tobias J. Bos ${ }^{1}$, Aladdin H. Hassanein ${ }^{1}$, Alison L. \\ Wong $^{2}$, Carol D. Morris ${ }^{3}$, Justin M. Sacks ${ }^{1}$

\begin{abstract}
${ }^{I}$ Department of Plastic and Reconstructive Surgery, Johns Hopkins University School of Medicine, Baltimore, MD 21205, USA.
${ }^{2}$ Department of Plastic and Reconstructive Surgery, Dalhousie University School of Medicine, Halifax, Nova Scotia B3H 4R2, Canada.

${ }^{3}$ Department of Orthopedic Surgery, Johns Hopkins University School of Medicine, Baltimore, MD 21205, USA.
\end{abstract}

\begin{abstract}
Correspondence to: Mr. Michelle Y. Seu, Department of Plastic and Reconstructive Surgery, Johns Hopkins University School of Medicine, Baltimore, MD 21205, USA. E-mail: mseu1@jhmi.edu
\end{abstract}

How to cite this article: Seu MY, Haley A, Cho BH, Carl HM, Bos TJ, Hassanein AH, Wong AL, Morris CD, Sacks JM. Proximal femur reconstruction using a vascularized fibular epiphysis within a cadaveric femoral allograft in a child with Ewing sarcoma: a case report. Plast Aesthet Res 2017;4:209-14.

Article history:

Received: 30 Jun 2017

First Decision: 26 Oct 2017

Revised: 6 Nov 2017

Accepted: 27 Nov 2017

Published: 30 Nov 2017

Key words:

Femur reconstruction,

vascularized fibula autograft,

Ewing sarcoma

\begin{abstract}
Periarticular reconstruction of appendicular bones in skeletally immature patients after tumor resection is a surgical challenge that requires a multidisciplinary approach. The authors present a case of Ewing sarcoma of the proximal femur in an 8-year old girl treated with wide resection of the primary tumor and reconstruction using a vascularized fibula epiphyseal autograft within a cadaveric femoral allograft. The native femoral head was preserved to restore articular anatomy. Postoperative course was without complications. This report demonstrates the use of a vascularized fibula autograft within a cadaveric femoral allograft to optimize growth potential and joint durability in a pediatric patient.
\end{abstract}

\section{INTRODUCTION}

Reconstruction of bones of the appendicular skeleton in pediatric patients poses a difficult challenge for reconstructive surgeons ${ }^{[1]}$. Treatment for Ewing sarcoma (ES), a common bone malignancy in individuals under the age of thirty years, often requires a complex, multi-disciplinary approach ${ }^{[1]}$. The current standard of care for localized ES combines surgical resection of the primary tumor with neoadjuvant and adjuvant chemotherapy to eliminate micrometastases and minimize the risk for recurrence ${ }^{[2]}$.

Complete surgical resection of the primary tumor and reconstruction using traditional prostheses often has good outcomes for adult patients ${ }^{[3,4]}$. However,

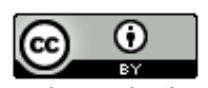
identical terms

For reprints contact: service@oaepublish.com

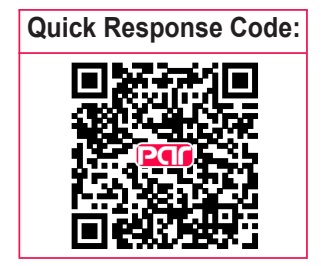

C) The author(s) 2017 


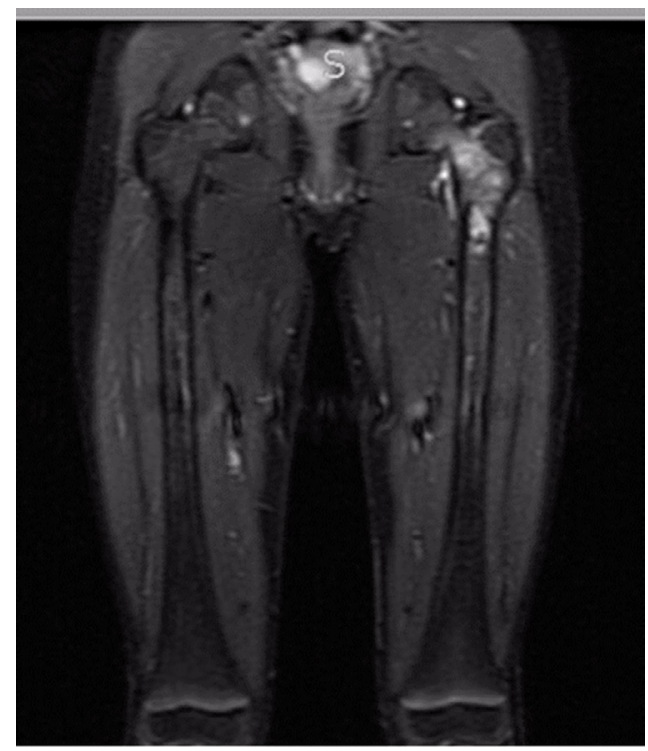

Figure 1: Magnetic resonance imaging of primary Ewing sarcoma tumor in patient's left hip

prostheses and non-vascularized allografts are insufficient for pediatric patients due to the need to both restore joint function and preserve growth potential ${ }^{[5]}$. Vascularized fibular head autografts have been previously used successfully in mandibular and radial reconstructions ${ }^{[6]}$. The proximal femur and acetabulofemoral joint has a unique structure that allows wide range of motion and weight-bearing properties. Reconstruction using a vascularized fibular autograft alone is insufficient to restore the native anatomy of the proximal femur. However, its structure can be augmented using an acellular cadaveric femoral allograft, which acts as a scaffold on which the autograft can grow ${ }^{[6]}$. Utilizing this method preserves the epiphyseal plate in a child, restoring both function and growth potential. Previous studies have combined acellular allografts with vascularized fibular autografts to reconstruct intercalary bony defects post-tumor resection ${ }^{[7-10]}$. However, there is a paucity of research on the reconstruction involving epiphyseal portions of long bones and articulations with this method ${ }^{[6]}$.

We present a case of an 8-year-old female with ES of the left proximal femur who underwent wide resection of the primary tumor followed by reconstruction of the acetabulofemoral joint using a vascularized fibular head free flap with a cadaveric femoral head allograft shell. The patient had no complications at 6-month follow-up. This case report demonstrates the novel use of an acellular cadaveric femoral allograft with a free fibula autograft including the articular head of the fibula for complex proximal femoral reconstruction of the femur and hip joint.

\section{CASE REPORT}

An otherwise healthy 8-year-old girl initially presented with left hip pain and was treated for presumed osteomyelitis; however, upon open biopsy of the left femoral neck, she was found to have ES extending from the femoral neck into the proximal diaphysis. The patient began a neoadjuvant chemotherapy regimen as specified by her local hospital protocol. Past medical history and family history are unremarkable.

Eight months after initial onset of symptoms, the patient and her family met with her treatment team at Johns Hopkins, which included pediatric orthopedic oncology and plastic and reconstructive surgery [Figure 1]. She and her family decided to pursue single staged resection of the primary tumor and reconstruction of the left proximal femur using an ipsilateral vascularized fibula autograft and cadaveric femoral allograft shell with articular restoration through preservation of the native femoral head.

The patient was taken to the operating room and placed on her right side. The left proximal femur was accessed via a lateral approach, starting proximal to the joint and extending distally approximately halfway down the thigh. The muscular attachments were dissected off the femur and the tumor was excised en bloc, revealing negative margins. An anterior capsulectomy was performed. The femur was dislocated from the acetabulum and a circumferential capsulotomy was performed in order to save the femoral head cap and preserve the native articular anatomy. After tumor excision, the defect extended from immediately below the femoral head cap to the proximal portion of the diaphysis.

The fibular harvest was initiated with a lateral incision on the ipsilateral leg. The musculature was elevated off the fibula and the anterior crural septum was transected. A distal osteotomy was performed $6 \mathrm{~cm}$ below the lateral malleolus using an oscillating saw. The deep peroneal artery and vein were ligated distally to be used as the vascular pedicle. The fibula and vascular pedicle were elevated completely out of the leg.

Within the left hip, the descending branch of the lateral femoral circumflex artery and vein were dissected out to their origin, prepared by microscope, and clamped. Meanwhile, the cadaveric femoral allograft shell was prepared ex-vivo by cutting the allograft length-wise and reaming out the core to create a trough for the fibular autograft. The fibular autograft was fractured to 

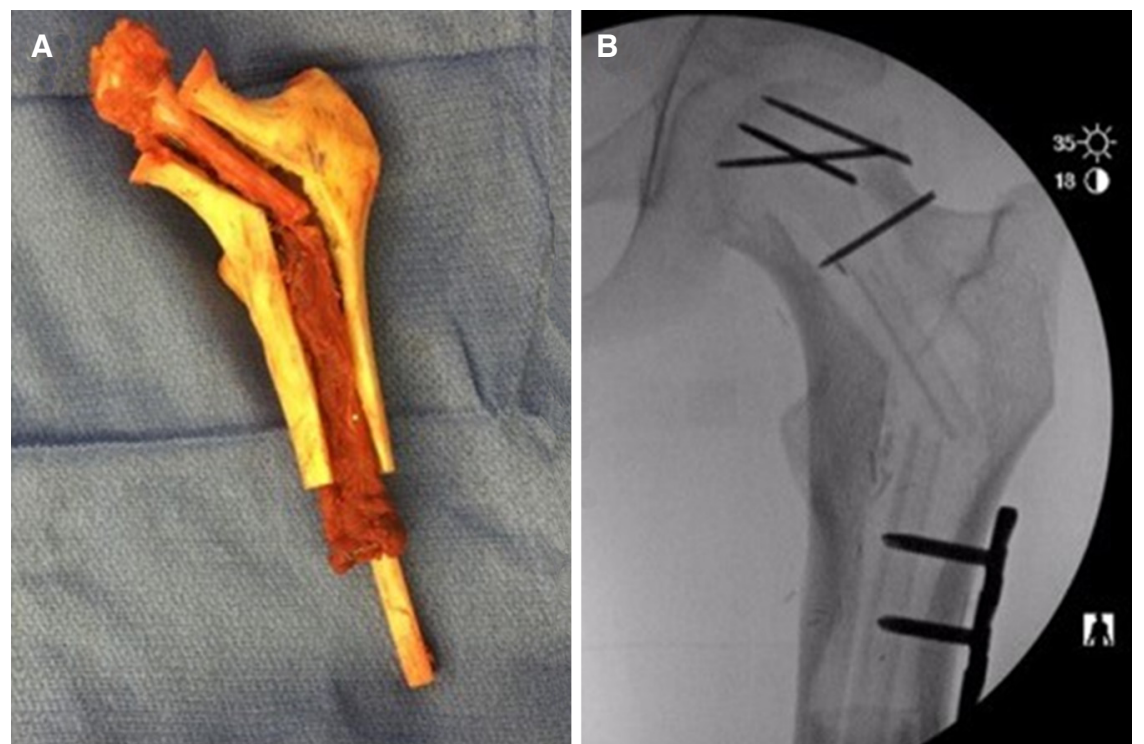

Figure 2: (A) Vascularized fibular autograft within cadaveric femoral allograft; (B) radiograph of final construct inset within patient's hip

recreate the angle of the femoral neck, then secured within the trough of the cadaveric femoral allograft shell. The fibula extended out of the distal portion of the allograft shell to ensure intussusception, or bony overlap within bone, into the native femoral diaphysis. The femoral allograft was reamed out to allow $1.5 \mathrm{~cm}$ of intussusception and the distal aspect of the autograft-allograft construct was malleted into the native femoral shaft. The native femoral head was modified to form a well-aligned cap to the proximal portion of the autograft-allograft complex. Terminally threaded Steinmann pins were used to hold the native femoral head cap and autograft-allograft complex together [Figure 2]. C-arm fluoroscopy was utilized to ensure that the pins did not protrude outside the bone. The left hip was reduced into the acetabulum and a 7-hole Synthes plate was placed to secure the autograft-allograft complex with the native femoral shaft. C-arm fluoroscopy was used again to ensure proper positioning of hardware in the construct.

The deep peroneal artery and vein were anastomosed in a retrograde fashion to the descending branch of the lateral femoral circumflex artery and vein [Figure 3]. A 2.5-mm coupler was used for venous anastomosis and 9-0 nylon suture was used to anastomose the arteries end-to-end. The patient was secured in a posterior splint and an epidural was placed in the operating room for postoperative pain management.

The patient tolerated the procedure well without perioperative surgical or anesthetic complications. She resumed her chemotherapy regimen immediately after surgery and returned to the operating room on postoperative day (POD) 7 for placement of a double leg spica cast for hip joint immobilization. Her postoperative stay was uneventful and she was transferred to her local hospital on POD 18 for continued care and management. She has had no complications at 6 months follow-up. Surveillance imaging has demonstrated good healing, maintenance of the hip joint, and absence of local recurrence. She remains non-weight bearing and will begin physical therapy after the completion of her chemotherapy regimen. At 9 months follow-up, the patient presented with no pain. X-rays demonstrated that the leg was healing well and that she could potentially advance her weight bearing status [Figure 4].

\section{DISCUSSION}

Advancements in skeletal reconstruction have improved options for limb salvage, but reconstruction remains challenging when tumors occur in the joints or epiphysis of children due to the need to preserve both growth and durability ${ }^{[7]}$. Most bone sarcomas are localized to the metaphysis of the bone. In adults, the entire proximal or distal portion of the bone is resected and reconstructed with prosthese ${ }^{[8]}$. Diaphyseal bone defects can be reconstructed using megaprostheses and intercalary allografts, both of which have high rates of postoperative complications such as nonunion, fracture, and infection ${ }^{[8-10]}$. However, the Capanna technique can also be considered as a method to reconstruct bony defects through the use of a vascularized autograft set within a decellularized allograft, and has demonstrated positive outcomes in variety of cases ${ }^{[11]}$. Though prostheses and allografts can successfully reconstruct joints in adults, special 


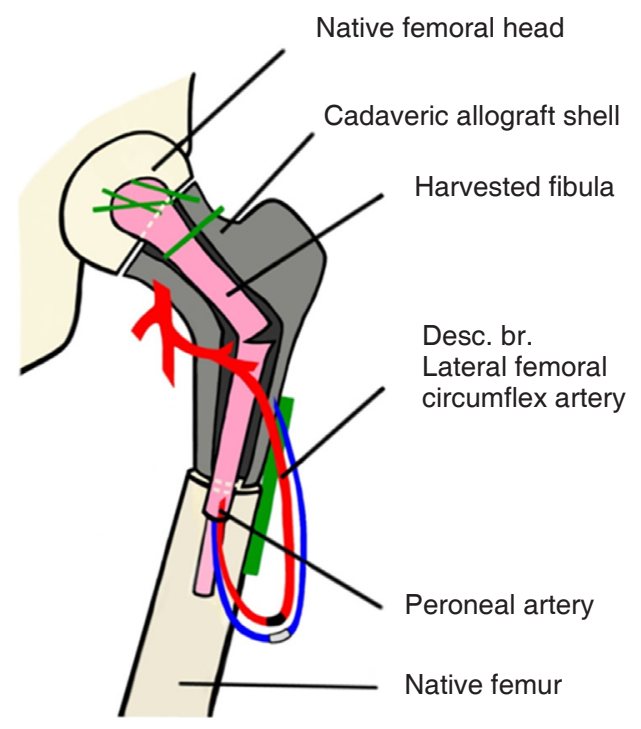

Figure 3: Schematic depicting final inset. Note intussusception of the distal portion of the fibula autograft into the femoral diaphysis

measures should be taken in pediatric patients to preserve growth potential, as well as restoration of a durable, functional joint that mimics native form.

Reports of using the Capanna technique in pediatric patients are limited ${ }^{[12]}$, however the decision to utilize this technique was pursued because it offered the patient the best prospective outcome in preservation of growth potential and durable joint function. The use of the decellularized cadaveric allograft in particular was opted because of its increased strength when compared to the fibula autograft alone ${ }^{[13]}$. Because the defect was extensive and required a long vascular pedicle, a larger portion of the autologous fibula than previously described by Capanna et al. ${ }^{[11]}$ was needed. The peroneal vessels provided the pedicle length necessary for anastomosis to the descending branch of the lateral femoral circumflex vessels. The epiphysis of the fibula was utilized to give the patient the best chance at continued growth, as previously described in similar procedures ${ }^{[14]}$. Because the peroneal artery is subject to atherosclerosis in adults, the anterior tibial artery is most often used as the vascular pedicle ${ }^{[15,16]}$. However, in children, atherosclerosis has typically not yet developed; thus, the peroneal artery is a good option in cases where increased pedicle length is needed.

This patient underwent neoadjuvant chemotherapy, which has been shown to be effective in shrinking tumor size and allowing a closer margin than would be considered safe in a tumor without this treatment ${ }^{[17,18]}$. It is known that vascularized fibular autografts are a preferred reconstruction material

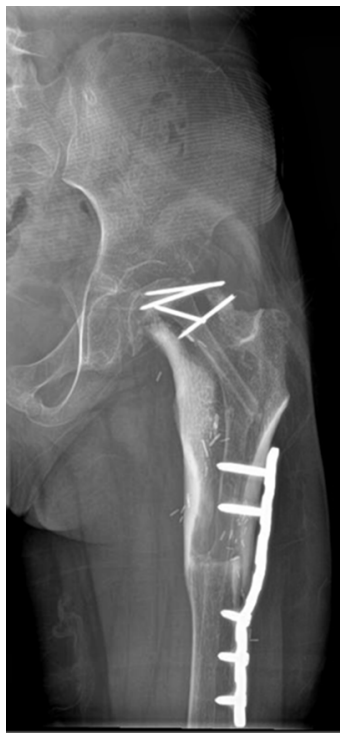

Figure 4: X-ray at 9 months postoperation indicating bony integration

due to their osteogenic capacity and resistance to infection, chemotherapy, and radiotherapy ${ }^{[5,19,20]}$. However, a fibular autograft alone would not provide the structural integrity needed to reconstruct the proximal femur and stabilize the hip joint ${ }^{[21]}$. The vascularized fibula within an allograft shell capitalizes on the immediate tensile strength of the allograft with the advantage of a biological autograft that can remodel and revascularize with the patient ${ }^{[8,11,15]}$. The patient's postoperative radiographs demonstrated bone healing and alignment and are likely to achieve complete union in the future.

The key to the success for this patient's reconstruction was the participation and cooperation of a multidisciplinary team in preoperative planning and throughout the surgery itself. Perspectives and techniques specific to orthopedic oncology, such as the design and inset of the final construct, as well as plastic and reconstructive surgery, including knowledge of lower extremity vasculature and technical skill in microsurgical anastomoses, were critical to providing the patient with the most appropriate treatment plan. Patients requiring complex skeletal reconstruction would benefit from seeking treatment at tertiary care centers where they have access to specialized surgical teams that can ensure that the patient is receiving personalized care.

The major contraindication for this technique is the inherently variant nature of vascular anatomy in the leg. Even in remarkably similar cases, surgeons should proceed with caution prior to surgery, making sure to utilize preoperative angiography in determining 
epiphyseal vascular supply ${ }^{[15]}$.

From this case report, we learned that single staged joint reconstruction is an option in joint reconstruction given comprehensive coordination between plastic surgery and orthopedic surgery teams. Though this is a rare case, it is a justifiable option in reconstruction of other joints, such as the shoulder and knee, where structure and function must be maintained. Discussion of the patient and their family's goals, such as preservation of the weight bearing nature growth potential of the limb, is crucial to patient selection. This is a long and technically complex procedure, and the ideal patient for this reconstruction is an otherwise healthy child with an extensive support network at a tertiary care center where collaboration between departments is possible.

In conclusion, this case report demonstrates the use of a vascularized fibula autograft within an acellular cadaveric allograft shell for complex reconstruction of the proximal femur and hip joint. The vascularized fibula autograft included the epiphyseal plate, allowing the reconstruction to grow with the patient. The cadaveric allograft shell serves as a biologic scaffold to allow the patient's vascularized free fibula with the fibular head to grow and expand into the construct through osteoconduction, providing increased stability to the reconstruction. This construct provides the potential for continued growth and weight bearing of the lower extremity. Patients requiring complex skeletal reconstructions should be treated at tertiary care centers where a multi-disciplinary team of orthopedic, oncologic, and reconstructive surgeons can be utilized.

\section{DECLARATIONS}

\section{Authors' contributions}

Concept and design: C.D. Morris, J.M. Sacks

Definition of intellectual content: B.H. Cho, A. Hassanein, A.L. Wong, C.D. Morris, J.M. Sacks

Manuscript preparation, data acquisition, literature search: M.Y. Seu, A. Haley

Manuscript editing: B.H. Cho, H.M. Carl, T.J. Bos, A.L. Wong

Manuscript review: A. Hassanein, C.D. Morris, J.M. Sacks

\section{Financial support and sponsorship} None.

\section{Conflicts of interest}

There are no conflicts of interest.

\section{Patient consent}

The patient's guardian provided us with consent to publish this case report.

\section{Ethics approval}

Obtaining ethical clearance was not necessary for the publication of this case report.

\section{REFERENCES}

1. Reed DR, Hayashi M, Wagner L, Binitie O, Steppan DA, Brohl AS, Shinohara ET, Bridge JA, Loeb DM, Borinstein SC, Isakoff MS. Treatment pathway of bone sarcoma in children, adolescents, and young adults. Cancer 2017;123:2206-18.

2. Weber KL. What's new in musculoskeletal oncology. J Bone Joint Surg Am 2005;87:1400-10.

3. Starc MT, Rosenblum MK, Meyers PA, Hatzoglou V. Rare presentation of Ewing sarcoma metastasis to the sella and suprasellar cistern. Clin Imaging 2016;41:73-7.

4. Yasko AW. Surgical management of primary bone sarcomas. Hematol Oncol Clin North Am 1995;9:719-31.

5. Houdek MT, Wagner ER, Stans AA, Shin AY, Bishop AT, Sim FH, Moran SL. What is the outcome of allograft and intramedullary free fibula (Capanna technique) in pediatric and adolescent patients with bone tumors? Clin Orthop Relat Res 2016;474:660-8.

6. Capanna RB, Campanacci M. A new technique for reconstructions of large metadiaphiseal bone defects. Orthop Traumatol 1993;3:159-77.

7. Dolen UC, Moran SL. Long term functional outcomes of the Capanna technique for pediatric limb salvage. Plast Reconstr Surg 2012;130:90-1.

8. Ogura M, Miyamoto S, Sakuraba M, Fujiwara T, Chuman H, Kawai A. Intercalary reconstruction after wide resection of malignant bone tumors of the lower extremity using a composite graft with a devitalized autograft and a vascularized fibula. Sarcoma 2015;2015:861575.

9. Muramatsu K, Ihara K, Tani Y, Iwanaga R, Taguchi T. Reconstruction of periacetabular bone tumor by vascularized fibula graft and irradiated autograft. Anticancer Res 2009;29:1669-73.

10. Berrey BH Jr, Lord CF, Gebhardt MC, Mankin HJ. Fractures of allografts. Frequency, treatment, and end-results. J Bone Joint Surg Am 1990;72:825-33.

11. Capanna R, Campanacci DA, Belot N, Beltrami G, Manfrini M, Innocenti M, Ceruso M. A new reconstructive technique for intercalary defects of long bones: the association of massive allograft with vascularized fibular autograft. Long-term results and comparison with alternative techniques. Orthop Clin North Am 2007;38:51-60.

12. Moran SL, Shin AY, Bishop AT. The use of massive bone allograft with intramedullary free fibular flap for limb salvage in a pediatric and adolescent population. Plast Reconstr Surg 2006;118:413-9.

13. Hariri A, Mascard E, Atlan F, Germain MA, Heming N, Dubousset JF, Wicart P. Free vascularised fibular graft for reconstruction of defects of the lower limb after resection of tumour. J Bone Joint Surg Br 2010;92:1574-9.

14. Manfrini M, Innocenti M, Ceruso M, Mercuri M. Original biological reconstruction of the hip in a 4-year-old girl. Lancet 2003;361:140-2.

15. Innocenti M, Delcroix L, Manfrini M, Ceruso M, Capanna R.Vascularized proximal fibular epiphyseal transfer for distal radial reconstruction: surgical technique. J Bone Joint Surg Am 2005;87:23746.

16. Martin AL, Bissell BM, Al-Dhamin A, Morris SF. Computed tomographic angiography for localization of the cutaneous perforators of the leg. Plast Reconstr Surg 2013;131:792-800.

17. Wafa H, Grimer R. Surgical options and outcomes in bone sarcoma. 
Expert Rev Anticancer Ther 2006;2:239-48.

18. Picci P, Sangiorgi L, Rougraff BT, Neff JR, Casadei R, Campanacci M. Relationship of chemotherapy-induced necrosis and surgical margins to local recurrence in osteosarcoma. J Clin Oncol 1994;12:2699-705.

19. Canosa R, Gonzalez del Pino J. Effect of methotrexate in the biology of free vascularized bone grafts. A comparative experimental study in the dog. Clin Orthop Relat Res 1994;301:291-301.
20. Taylor GI, Miller GD, Ham FJ. The free vascularized bone graft. A clinical extension of microvascular techniques. Plast Reconstr Surg 1975;55:533-44

21. Abed YY, Beltrami G, Campanacci DA, Innocenti M, Scoccianti G, Capanna R. Biological reconstruction after resection of bone tumours around the knee: long-term follow-up. $J$ Bone Joint Surg Br 2009;91:1366-72 